\title{
Evaluación de la Faceta Epistémica del Conocimiento Didáctico- Matemático de Futuros Profesores de Matemáticas en el Desarrollo de una Clase Utilizando Funciones
}

\author{
Evaluation of the Epistemic Facet of Didactic-Mathematical Knowledge of \\ Future Mathematics Teachers in a Class Development Using Functions
}

\author{
Tulio Amaya De Armas* \\ ORCID iD 0000-0003-0342-4338
}

\begin{abstract}
Resumen
Se reportan los hallazgos de un trabajo realizado con cincuenta futuros profesores de licenciatura en matemática de una universidad pública colombiana. A partir de un seguimiento en su progreso de estudiantes a profesores, se analizaron sus dificultades en el desarrollo de clases con estudiantes de enseñanza media. Los resultados evidencian cierta facilidad en los futuros profesores en el reconocimiento de los elementos y las características de una función, pero algunas dificultades para conectar sus representaciones. Por una parte, utilizaron materiales didácticos y representaciones semióticas adecuadas, y por otra, realizaron pocas congruencias entre elementos de éstas y no establecieron conexiones con elementos del contexto sociocultural que les permitiera un análisis a profundidad del tema. Con relación a sus conocimientos, se evidencian ciertas fortalezas en su conocimiento común y ampliado, pero algunas dificultades en el conocimiento especializado del contenido.
\end{abstract}

Palabras clave: Profesores en formación. Faceta epistémica. Conocimiento didáctico-matemático: Funciones. Representaciones semióticas.

\begin{abstract}
We report here the findings of a work done with fifty preservice teachers, students of mathematics from a Colombian public university. From a follow-up on the progress of students to teachers, we analyzed their difficulties in class development with high school students. The results show ease for the preservice teachers in the recognition of the elements and characteristics of a function, but certain difficulties to connect the elements of their representations. On the one hand, they used didactic materials and adequate semiotic representations, and on the other, they made few congruences between these elements and did not establish connections with elements of the sociocultural context that would allow an in-depth topic analysis. Regarding their knowledge, certain strengths are evidenced in their common and extended knowledge, but there are some difficulties in specialized content knowledge.
\end{abstract}

Keywords: Preservice teachers. Epistemic facet. Mathematical didactic knowledge. Functions. Semiotic representations.

\footnotetext{
* Doctor en Innovación e Investigación en Didáctica por la Universidad Nacional de Educación a Distancia de España (UNED). Académico de la Facultad de Educación de la Universidad Católica de la Santísima Concepción (UCSC), Alonso de Ribera, 2850, Chile. Dirección postal: Calle 38, n. 16 A-26 Sincelejo, Colombia. Email: tuama1@hotmail.com.
} 


\section{Introducción}

El trabajo con funciones resulta problemático tanto en los procesos de enseñanza como en los de aprendizaje, esto a pesar de que es uno de los conceptos matemáticos que admite mayor cantidad de registros en los que es posible representarlo (HITT; MORASSE, 2009; DOLORES, 2013) y a la relativa facilidad en la producción de sus diversas representaciones. Según Even (1990) el estudio de las funciones precisa del análisis de sus diversas representaciones, por lo que el estudiante debe poder pasar de una representación a otra sin caer en contradicciones, y hacer conexiones entre ellas y con elementos del contexto sociocultural, para poder comprenderlas.

Haciomeroglu (2006) resalta que la multidimensionalidad de las funciones representa un desafío, tanto para profesores en formación como para profesores experimentados, ya que para traducir entre las diversas formas de representaciones de una función es necesario tener una visión bien fundamentada de la forma en que su definición se relaciona con cada representación. Dicha visión requiere de un profesor con una amplia y variada interacción con el concepto, con una buena comprensión matemática, así como de las cuestiones pedagógicas pertinentes, además del conocimiento de aspectos curriculares y socioculturales que afectan el aprendizaje de los estudiantes.

Dicho de otra manera, se trata de que "Para que un profesor llegue a entender qué es lo que comprende un alumno requiere comprender profundamente el material que enseña y la dinámica de los procesos de aprendizaje de los estudiantes" (SHULMAN, 2005, p.25).

Even (1990) y Haciomeroglu (2006) consideran que el conocimiento sobre funciones, por parte de futuros maestros, es débil y frágil, encontrando en ellos serias dificultades para producir y relacionar sus representaciones. Mientras Rey et al. (2009) manifiestan que, con relación al proceso de enseñanza y aprendizaje de las funciones, se acostumbra a proponer actividades de conversión de la representación algebraica a otras representaciones, pero es poco frecuente que se consideren conversiones desde otras representaciones hacia la algebraica. Por otra parte, en su enseñanza, no se promueve el análisis de situaciones en condiciones de variación y cambio, donde se facilite asignar significado y sentido a las funciones analizadas.

De lo planteado por Even, (1990) y Haciomeroglu (2006) se puede inferir que el conocimiento didáctico-matemático sobre funciones, de los profesores en formación, no parece estar bien fundamentado, de forma que les permita orientar eficientemente un proceso de aprendizaje que las incluyan. Así mismo, en lo planteado por Rey et al. (2009), que el estudio 
que se hace de las funciones no permite analizar la relación entre sus elementos, ni articular sus diversas representaciones.

Según Villa-Ochoa (2015) el estudio de las funciones, sin articular los elementos de sus representaciones, lleva a una falta de conexión entre dichas representaciones, lo cual no permite un estudio integral de este concepto. Esta falta de articulación puede resultar problemática, ya que, al no hacerse un análisis de congruencias e incongruencias entre un número considerable de las representaciones de un objeto matemático, lleva a entenderlas como algo separado del objeto estudiado y, también, puede implicar que el aprendiz confunda la representación con el objeto representado (DUVAL, 2017).

Las investigaciones anteriores, junto a otras, han ido generando cierto consenso en la comunidad de personas interesadas en la mejora de la enseñanza de las funciones, sobre la necesidad de enseñarlas teniendo en cuenta sus diferentes representaciones y los tratamientos y conversiones entre ellas. Se trata de una orientación para guiar la práctica del profesor que se ha generalizado para cualquier objeto matemático más allá de las funciones. De hecho, esta orientación (presentar una muestra representativa e interconectada de las diferentes representaciones de un objeto matemático) está contemplada en el criterio de idoneidad epistémica, uno de los seis criterios que conforman la noción de idoneidad didáctica de un proceso de instrucción (BREDA; PINO-FAN; FONT, 2017; BORJI; SÁNCHEZ, 2019).

Dicho criterio (presentar una muestra representativa e interconectada de las diferentes representaciones de un objeto matemático) se pueden interpretar como una creencia del profesor, si entendemos, de acuerdo con Peirce (1877), la creencia como una disposición para la acción. Ahora bien, tanto para generar esta creencia como para guiar la acción con base a esta creencia el futuro profesor necesita ciertos conocimientos (ERTMER; OTTENBREITLEFTWICH, 2010). En particular, conocimientos de la faceta epistémica del conocimiento didáctico-matemático del modelo de competencias y conocimientos didáctico-matemáticos del profesor de matemáticas (modelo CCDM) (GODINO et al., 2017).

De acuerdo con lo expuesto anteriormente, esta investigación tuvo como objetivo evaluar la faceta epistémica del conocimiento didáctico-matemático de profesores en formación al hacer transformaciones de las representaciones de una función. Para ello, se puso el foco del análisis en los múltiples registros y representaciones de algunas relaciones funcionales y se enfatizó en el análisis de los sistemas semióticos emergentes que se logran producir. 


\section{Aproximaciones teóricas}

\subsection{Conocimiento didáctico matemático}

En las últimas décadas, las investigaciones en Educación Matemática han tenido un importante desarrollo en cuanto a los conocimientos que debe dominar un profesor de matemáticas, y, a partir de ello, se han propuesto múltiples modelos (SHULMAN, 1997, 2005; GROSSMAN, 1990; BALL y sus colaboradores (BALL; HILL; BASS, 2005; BALL; THAMES; PHELPS, 2008; HILL; BALL; SCHILLING, 2008); SCHOENFELD; KILPATRICK, 2008) y GODINO y sus colaboradores (GODINO; BATANERO; FONT, 2007; GODINO, 2009; PINO-FAN; GODINO, 2015; PINO-FAN; ASSIS; CASTRO, 2015)), tratando de facilitar el análisis de los elementos distintivos de los conocimientos de un profesor de matemáticas. Pero a pesar de los avances logrados en dichos estudios, "los modelos de conocimiento matemático para la enseñanza elaborados desde las investigaciones en educación matemática, incluyen categorías demasiado globales y disjuntas" (GODINO, 2009, p. 19), que no permiten un análisis en profundidad de los conocimientos matemáticos y didácticos de un profesor.

Dado que el análisis del conocimiento didáctico-matemático de un grupo de profesores en formación podría resultar demasiado extenso, por los diferentes conocimientos (BALL; THAMES; PHELPS, 2008), facetas (SHULMAN, 1997; GODINO, 2009) o dimensiones (SCHOENFELD; KILPATRICK, 2008) que habría que analizar, en esta investigación se enfatiza solo el análisis de la faceta epistémica (GODINO, 2009; PINO-FAN; GODINO, 2015) o conocimiento relativo al contenido matemático (BALL; THAMES; PHELPS, 2008; HILL; BALL; SCHILLING, 2008). La faceta epistémica está compuesta por elementos de dos dimensiones: la dimensión matemática en su totalidad (Conocimiento común y ampliado) y uno de la dimensión didáctica (el conocimiento especializado del contenido).

El conocimiento común del contenido (denominado por Ball y colaboradores Common Content Knowledge o CCK) es el que un profesor comparte con los estudiantes en el nivel donde enseña, y le sirve para escoger y resolver tareas adecuándolas a las posibilidades de comprensión de los estudiantes con los que trabaje. El conocimiento ampliado del contenido (Denominado por Ball y colaboradores Horizon Content knowledge o HCK) es el conocimiento que le permite al profesor producir y conectar diversas representaciones de un objeto matemático, resolver tareas utilizando distintos procedimientos, hacer generalizaciones, vincular objetos matemáticos de diferentes niveles educativos, tanto anteriores como 
posteriores, con los objetos del nivel donde se enseña (PINO-FAN; GODINO, 2015).

El conocimiento especializado del contenido (denominado por Ball y colaboradores Specialized Content Knowledge o SCK) es, según Ball, Thames y Phelps (2008) el conocimiento matemático que le permite al profesor saber cómo enseñar. Mientras que para Pino-Fan; Godino (2015) el SCK es un conocimiento de la dimensión didáctica que habilita al profesor para hacer transposiciones didácticas adecuadas a quien se enseña, que los lleven a movilizar y comprender la diversidad de significados parciales para un objeto matemático en los diversos contextos en donde sea posible analizarlo. Según estos autores, el SCK, además, debe habilitar al profesor para hacer diversas justificaciones y argumentaciones e identificar los conocimientos puestos en juego durante el desarrollo de una tarea matemática.

Más que consensuar a qué dominio pertenece este conocimiento, lo que interesa es que el SCK le permite al profesor gestionar y escoger los recursos y registros apropiados para el desarrollo de la clase, y la forma en que los objetos matemáticos, a estudiar, se presentan a los estudiantes. Así mismo, habilita al profesor para reproducir las representaciones de los objetos estudiados y para establecer conexiones entre sus diferentes elementos, que permitan relacionarlos con elementos del contexto sociocultural, donde al estudiante se le facilite asignarle significados y sentidos. Los tres conocimientos en su conjunto son los facilitadores para la clase de matemáticas, y son factores determinantes al momento de generar mayor posibilidad de éxito en los aprendices.

En lo planteado anteriormente, se puede inferir que un profesor debe conocer las matemáticas a profundidad (EVEN, 1990) y poder armonizar sus conocimientos didácticomatemáticos en beneficio del aprendiz. La armonización implica una conexión total entre los elementos necesarios para poner en funcionamiento óptimo los procesos cognitivos que lleven a la noesis (DUVAL, 2004), y se conjuga cuando el docente articula sus conocimientos didáctico-matemáticos, poniéndolos al servicio del funcionamiento de la clase.

Esta articulación le permite al profesor relacionar los contenidos que orienta con conocimientos básicos de otros niveles, tanto inferiores como posteriores, es decir, identificar las necesidades del aprendiz para comprender el tema y proyecta lo que orienta hacia un futuro académico del estudiante. Además, escoge recursos adecuados para el desarrollo de la clase y establece conexiones entre los elementos del mayor número de representaciones producidas y de éstas con las representaciones fenomenológicas asociadas.

Como complemento a lo anterior, se puede decir que un profesor que posee un conocimiento especializado, bien fundamentado, toma los elementos del conocimiento común para compartirlos con sus estudiantes, para ello requiere del conocimiento ampliado del 
contenido e ir a niveles anteriores y revisar los requerimientos que se necesitan para que los estudiantes le entiendan. Además, es necesario que pueda proyectarse a niveles posteriores y fundamentarse en conocimientos matemáticos avanzados que le permitan asegurarse que lo que se trabaja coincide con lo institucionalizado y que, a futuro será de utilidad a quien aprende.

Los conocimientos común, ampliado y especializado del contenido son indispensables en un profesor de matemáticas y están muy relacionados entre sí, cada uno cumple funciones específicas, pero no exclusivas, ya que en algunos casos las comparten (AMAYA; PINO- FAN; MEDINA, 2016). Es en las funciones compartidas y superposiciones entre los conocimientos común, ampliado y especializado del contenido, donde está el fundamento para la armonización de los contenidos que se orientan.

El análisis y caracterización de los objetos matemáticos primarios (lenguajes o representaciones, situaciones problema, procedimientos/estrategias, conceptos/definiciones y argumentos) y procesos presentes en las prácticas matemáticas que desarrollan los profesores en el desarrollo de su actividad docente, según Pino-Fan, Assis y Castro, (2015), se hace a través de la noción de configuración ontosemiótica (formada por los problemas, prácticas, objetos y procesos matemáticos). La noción de configuración ontosemiótica aporta criterios para delimitar subcategorías de conocimientos didáctico-matemáticos y permite identificar y describir en detalle los objetos en estudio, sus significados, así como los procesos desarrollados en las prácticas matemáticas, tanto institucionales como personales.

\subsection{Funciones}

El objeto de estudio en esta investigación, desde el saber disciplinar, para analizar el estado de desarrollo de la faceta epistémica en los profesores en formación, son las funciones. Si bien "Una función $f$ es un conjunto de pares ordenados $(x, y)$ ninguno de los cuales tienen el mismo primer elemento" (APÓSTOL, 1985, p. 65), el objeto matemático función es el resultado de una emergencia que se ha producido a lo largo de mucho tiempo. La evolución histórica de la noción de función muestra como a lo largo de la historia dicha noción se ha entendido de maneras diferentes. En cada una de estas maneras de entender se utilizan propiedades, representaciones definiciones y argumentos diferentes, y se resuelven tipos de problemas diferentes (configuraciones ontosemióticas). En particular, la evolución histórica de la noción de función muestra que, a lo largo del tiempo, se han utilizado diferentes representaciones para dicha noción. 
La función es uno de los objetos matemáticos que facilita hacer un mayor número de representaciones: sagitales, tabulares, gráficas, fenomenológicas, cartesianas y analíticas: estás últimas pueden ser de dos tipos: analítico numéricas y analítico algebraicas. Así mismo, como elementos que permiten un estudio contextualizado del objeto matemático función, están las relaciones funcionales. Una relación funcional es una función identificable en un contexto concreto determinado. Una de las posibles maneras de concebir el significado de un objeto matemático, en nuestro caso función, es considerar que dicho significado es su definición. Esta manera de entender el significado es una forma elemental o unitaria, y otra posibilidad de afrontar el problema del significado es hacerlo en términos de su uso (GODINO; BATANERO, 1994).

Desde esta perspectiva el significado de un objeto matemático se debe entender en términos de lo que se puede hacer con dicho objeto. Se trata de una perspectiva pragmatista y sistémica, ya que se considera que el significado de un objeto es el conjunto de prácticas en las que dicho objeto es determinante para su realización. Dicho conjunto de prácticas se puede compartimentar en diferentes significados parciales, el conjunto de los cuales se considera el significado global o de referencia de la noción de función.

Cuando se utilizan las funciones en las prácticas matemáticas, además de su definición, se utilizan diferentes representaciones, así como determinadas características y propiedades, y otros objetos matemáticos relacionados como son las ecuaciones etc. Una buena herramienta para mostrar esta complejidad es la herramienta configuración ontosemiótica, la cual permite concretar la noción de significado parcial en términos de prácticas y de configuraciones ontosemióticas activadas en dichas prácticas.

En general, el objeto matemático función es, sin lugar a dudas, un elemento fundamental del desarrollo histórico de la humanidad. Ha sido ampliamente estudiado, lo que ha permitido establecer algunos significados institucionales o epistémicos de referencia, materializados en los contenidos matemáticos de los planes de estudio, los programas de asignaturas y los significados que se les han dado en los libros de textos (BUENO; PÉREZ, 2018; PINO-FAN, 2013). A lo largo de su desarrollo histórico, la noción función ha sido asociada con, por lo menos, seis significados parciales (PINO-FAN; PARRA; CASTRO, 2019), como: correspondencia entre magnitudes; relación entre magnitudes variables, representación gráfica, expresión analítica, correspondencia arbitraria y como relación entre los elementos de dos conjuntos. 


\subsubsection{Conflictos epistémicos asociados al concepto de función}

En un proceso de estudio que involucre funciones, la diferencia de significados entre una representación y otra, genera conflictos, que obstaculizan la comprensión del concepto (ROJAS, 2015). El estudio de situaciones problemas que involucran relaciones funcionales, en diferentes periodos históricos, así como los invariantes colectivos y las distintas representaciones semióticas utilizadas, han permitido distinguir diferentes conflictos epistémicos asociados a la noción de función.

Los conflictos epistémicos son desajustes entre lo pretendido institucionalmente, lo implementado por el docente en su clase y lo realmente comprendido por el aprendiz (GODINO; BENCOMO; WILHELMI, 2006), por lo que es necesario su análisis por parte de quien orienta el proceso de enseñanza, para que los desajustes en el proceso de aprendizaje sean utilizados en beneficio de quien aprende y puedan minimizarse. Además, para que los conflictos epistémicos sean útiles en el proceso, es necesario que el mismo docente pueda producir y analizar los elementos matemáticos primarios del objeto matemático que estudia. Entre los conflictos epistémicos que se han podido aislar en el trabajo con funciones están:

\section{$>\quad$ El desconocimiento de la letra como variable}

Este es un conflicto muy común en contextos académicos, que es donde es más identificable. Consiste en la no aceptación de la letra como una cantidad susceptible de cambios $\mathrm{y}$, por tanto, no se reflexiona sobre la cuantificación de dichos cambios, por lo que se ha convertido en un gran obstáculo en el aprendizaje de procesos algebraicos (FONT, 2011), lo que según Hitt (2003), podría ser un factor que impida el acceso al cálculo.

La no aceptación de representaciones diferentes a la analítica como representación de una función

Es un conflicto que se da prácticamente a todos los niveles académicos, incluso hasta en los profesores universitarios. Consiste en que la única representación que reconocen para una función es la aritmético numérica o la aritmético algebraica, materializada en una fórmula, o un polinomio aritmético. No aceptan una tabla, una gráfica, un diagrama, una secuencia numérica, ni un conjunto de parejas ordenadas como representaciones de una función, y por tanto no los usan como apoyo para dar respuestas a cuestiones por las que se les indaguen, aunque los hayan reproducido previamente (AMAYA; PINO- FAN; MEDINA, 2016). Este conflicto genera una dificultad en la integración de los distintos registros y representaciones (DOLORES, 2013), por lo que no permite que se haga un análisis integral del sistema semiótico producido. Además, no conciben una relación funcional como una función. 


\section{$>\quad$ Confundir la letra como magnitud con la letra como variable generalizada}

Es otro conflicto con las características del descrito anteriormente, se da prácticamente en todos los niveles académicos. Ambos conflictos, el anterior y este, son heredables, es decir, se vienen transmitiendo de generación en generación, por tanto, su origen parece ser epistemológico-didáctico. Este se da al modelar relaciones funcionales con expresiones algebraicas del tipo $e=v t$ - $g t^{2} / 2$ (donde $e$ representa el espacio recorrido por un móvil que se lanza al espacio, $v$ la velocidad, g la gravedad en ese lugar y $t$ el tiempo en que lo recorre) y considerarla equivalente a la fórmula matemática $y=a x-b x^{2} / 2$, se está haciendo el paso de las magnitudes concretas- de espacio y tiempo- a las variables generales ( $x$ e $y$ ) (FONT, 2011).

La minimización de estos conflictos es de suma importancia en el aprendizaje del cálculo, teniendo en cuenta que la identificación y uso de elementos cambiantes, al analizar procesos de variación y cambio, son elementos esenciales que pueden conducir a la determinación de la variable (HITT; MORASSE, 2009).

La identificación y uso de secuencias, tablas de valores, el análisis de regularidades y patrones, implica la presencia de ciertos rasgos de funcionalidad (REY et al., 2009). Los rasgos de funcionalidad se relacionan con aquellos fenómenos que pueden cambiar, continua y constantemente, con diferentes grados de intensidad entre unos límites determinados y lo que en ellos se estudia, son magnitudes variables que llevan consigo alguna potencialidad de ser medidas.

La pluralidad de significados parciales del objeto función, cada uno de los cuales se relaciona con una o varias representaciones de ésta y con los conflictos epistémicos que pueden implicar su estudio, conllevan que el profesor deba tener un amplio conocimiento didáctico matemático de esta noción. En particular, tener bien fundamentada su faceta epistémica, para desarrollar una enseñanza idónea con sus alumnos, en la que las diferentes representaciones de la función y las conversiones y los tratamientos, que entre ellas se logren realizar, tengan un papel fundamental.

\subsection{Las representaciones semióticas}

Las representaciones semióticas, según Duval (2017), son construcciones por medio de signos con las que los individuos exteriorizan sus representaciones mentales. Los registros semióticos son los contenedores donde se reproducen las representaciones. Cada registro tiene sus propias reglas (DUVAL, 2004), pero éstas no son exclusivas, en algunos casos son compartidas por diferentes registros. Así mismo, llamamos sistema semiótico emergente al 
conjunto de registros y representaciones que se puedan producir de un objeto matemático identificable en un contexto determinado.

Las representaciones semióticas permiten dos tipos de transformaciones: las conversiones, que son transformaciones que se hacen en las representaciones cambiando de registro, y los tratamientos, que son transformaciones que se hacen sin cambiar de registro. Así mismo, es posible identificar algunos elementos en varias representaciones de un mismo objeto (establecer congruencias). Cuando es posible establecer congruencias entre dos o más representaciones, se dice que éstas son homogéneas, sino, se dice que son heterogéneas. "Este análisis de congruencias es un fuerte potencializador para la comprensión de las funciones, al hacer el tránsito entre todas las representaciones posibles y relacionarlas con los elementos de las representaciones del registro fenomenológico" (AMAYA; PINO- FAN; MEDINA, 2016, p. $113)$.

El análisis de los sistemas semióticos emergente es de tal importancia que Duval (2004) considera que para que haya aprendizaje matemático se requiere que el que aprende logre conectar por lo menos dos registros semióticos de representación del objeto matemático estudiado. Además, todo progreso de conocimiento en matemáticas pasa por este trabajo de transformación y análisis; por lo que son prioritarias las posibilidades de transformar una representación semiótica en otra representación semiótica, como base en un proceso de comunicación que busca saber cómo puede ser codificado/decodificado un objeto matemático para poder ser comprendido por alguien.

\section{Aspectos metodológicos}

\subsection{Tipo de estudio}

Es un estudio eminentemente cualitativo, donde se interactuó con estudiantes de licenciatura en matemáticas, se observaron clases, se analizaron posibles dificultades con la solución de las tareas que se planeaban para proponer a los estudiantes de la enseñanza media y se discutían posibles alternativas para minimizar las dificultades que se encontrarían. Se centró la atención en los conocimientos y habilidades que un profesor de matemáticas necesita para lograr una instrucción que facilite, de la mejor manera, el aprendizaje de los estudiantes.

Es un estudio exploratorio donde se analizan las configuraciones cognitivas que emergen de los profesores en formación al dar sus respuestas a las cuestiones por las que se indagaron en este proceso. Esta variable, según Pino-Fan (2013), está íntimamente relacionada 
con el tipo de conocimiento didáctico-matemático que poseen los profesores en formación.

El análisis se plantea desde lo institucional (configuraciones epistémicas), es decir, de los conocimientos esperados, así como desde lo personal (configuraciones cognitivas), al analizar los conocimientos efectivamente logrados por los profesores en formación. Se buscó describir las respuestas de los profesores en formación ante las demandas de la enseñanza del día a día, momento a momento. En este sentido, el conocimiento se produce como resultado de la interacción de los profesores en formación con los demás elementos del proceso formativo.

\subsection{Muestra de informantes}

La muestra de informantes, en esta investigación la constituyen veinticuatro profesores en formación del sexto semestre de un programa de licenciatura en matemáticas y veintiséis que habían cursado el programa en su totalidad y solo cursaban la práctica docente. Los grupos se eligieron de forma intencional, utilizando como criterio de inclusión que los participantes hubieran cursado nueve asignaturas que se ofrecen en el programa: Prácticas Pedagógicas Investigativas (PPI: se ofrecen de la I a la V) y cuatro Didácticas de las Matemáticas (DIMES: de la I a la IV).

Las PPI y DIMES que se ofrecen en el programa son asignaturas que se comienzan a ofrecer desde el segundo semestre y se continúan ofreciendo ininterrumpidamente hasta el sexto semestre. Los profesores en formación de la muestra tenían edades entre 20 y 27 años. El programa licenciatura en Matemáticas tiene una duración de ocho semestres.

\subsection{Recolección de la información}

A los profesores en formación se les pidió buscar, en revistas, libros de texto o por internet, algunas actividades que involucraran funciones, para el desarrollo de sus clases durante el semestre. Además, para la búsqueda, se les sugirió un material clasificado por Godino, Batanero y Font (2003), y también se les entregó el enunciado de diez situaciones.

Del material disponible se escogieron seis situaciones, se hicieron algunos ajustes, y se acordó que todos debían trabajar las mismas, las cuales fueron validadas según criterio de expertos. Aquí se reportan los resultados del desarrollo de una sola de dichas actividades, en la que, a partir de una hoja tamaño carta, debían construir una caja sin tapa y preparar una clase, que, posteriormente, desarrollarían ante estudiantes de enseñanza media. 
A pesar que la situación seleccionada se refiere a la elaboración de un empaque, y que el sistema de empaquetamiento en la actualidad ha cambiado mucho por el uso de la tecnología, se considera que es una actividad muy relevante con fines didácticos, puesto que permite reproducir múltiples representaciones con un alto grado de homogeneidad, que facilita conectar sus elementos y asignarles significado, al articularlos con elementos del contexto sociocultural.

Este tipo de actividades, según Shulman (2005), revela e ilumina los complejos cuerpos de conocimientos y habilidades que se necesitan para que un profesor sea competente. En este sentido, se esperaba que, la combinación de procesos interactivos, entre los profesores en formación con los estudiantes de enseñanza media, a través del uso de diferentes estrategias de solución, y recursos didácticos, en el desarrollo de las clases, facilitara la valoración del conocimiento del contenido en relación con la enseñanza (PINO-FAN, 2013).

A continuación, se muestran en el Cuadro 1, las categorías y los indicadores que se tuvieron en cuenta para el análisis de la actividad desarrollada por los profesores en formación en sus clases ante estudiantes de enseñanza media.

\begin{tabular}{|c|c|}
\hline Categoría de análisis & Indicadores \\
\hline Soluciona la tarea propuesta & Procedimientos adecuados al nivel de los estudiantes \\
\hline $\begin{array}{l}\text { Reconocimiento y uso de los elementos de } \\
\text { una función }\end{array}$ & $\begin{array}{l}\text { Determinación de: } \\
\text { a) Los intervalos de variación } \\
\text { b) valores extremos } \\
\text { c) intervalos de crecimiento/decrecimiento }\end{array}$ \\
\hline Producción de registros y representaciones & $\begin{array}{l}\text { Registros de partida y auxiliares usados y } \\
\text { representaciones producidas }\end{array}$ \\
\hline $\begin{array}{l}\text { Análisis de los sistemas semióticos } \\
\text { emergentes producidos }\end{array}$ & $\begin{array}{l}\text { Conexiones realizadas entre registros y } \\
\text { representaciones }\end{array}$ \\
\hline $\begin{array}{l}\text { Uso de diferentes procedimientos o } \\
\text { estrategias para resolver una tarea }\end{array}$ & $\begin{array}{l}\text { Tipo de estrategia usada y articulación entre las } \\
\text { soluciones } \\
\text { Identifica posibles casos generales } \\
\text { Pertinencia entre los recursos usados con la tarea } \\
\text { desarrollada }\end{array}$ \\
\hline $\begin{array}{l}\text { Uso de proposiciones/definiciones en la } \\
\text { solución de una tarea }\end{array}$ & $\begin{array}{l}\text { Pertinencia de la definición con los elementos } \\
\text { analizados } \\
\text { Propone nuevas tareas que comprometan al } \\
\text { estudiante en su propio proceso de aprendizaje y } \\
\text { evidencien la articulación del temas, con temas } \\
\text { anteriores y con otros más avanzados o con otros } \\
\text { temas del currículo }\end{array}$ \\
\hline $\begin{array}{l}\text { Argumentos utilizados para comunicar una } \\
\text { idea }\end{array}$ & $\begin{array}{l}\text { Calidad de las explicaciones y justificaciones de los } \\
\text { procesos realizados }\end{array}$ \\
\hline
\end{tabular}

Cuadro 1 - Elementos para el análisis de la faceta epistémica

Fuente: elaboración propia

La información se recogió en el lugar de desempeño habitual de los sujetos investigados, en diferentes momentos durante todo el semestre académico 02-2016. Los datos recogidos consisten en las transcripciones de entrevistas y videograbaciones, y notas producto de la observación recogidos en el trabajo de campo. 


\subsection{Tratamiento y análisis de la información}

A partir de las clases desarrolladas se analizaron y caracterizaron los elementos matemáticos primarios y aquellos procesos presentes en las prácticas matemáticas que desarrollaron los profesores en formación. Para identificar y describir la actividad matemática desarrollada por los profesores en formación en el desarrollo de su clase, se utilizó la noción de configuración ontosemiótica (PINO-FAN; ASSIS; CASTRO, 2015).

Aquí se reportan los resultados solo de la faceta epistémica. En la presentación de los resultados, para cada ítem se presenta una dupla $(i, \mathrm{j}$ ) donde $i$ (entre 0 y 24 corresponde a estudiantes del sexto semestre) y $j$ (entre 0 y 26 son estudiantes del octavo semestre) representa la cantidad de profesores en formación que hicieron referencia al tópico analizado en ese ítem. De la misma manera, se presenta $\mathrm{P}_{(\mathrm{r}) \mathrm{i}, \mathrm{j}}$, donde $\mathrm{r}=6 \mathrm{u} 8$ semestre, para referir a un profesor en formación de alguno de los dos niveles al que se hace referencia de su respuesta.

\section{Evaluación de la faceta epistémica, del conocimiento de profesores en formación desarrollando una clase}

Para el desarrollo de la clase, a los profesores en formación se les propuso utilizar la siguiente situación, con esas orientaciones:

Situación:

Construir una caja sin tapa con una hoja de papel tamaño carta $(21,8 \mathrm{~cm} \times 28,7 \mathrm{~cm})$, al quitar en las esquinas cuadraditos de lado l (ver Figura 1).

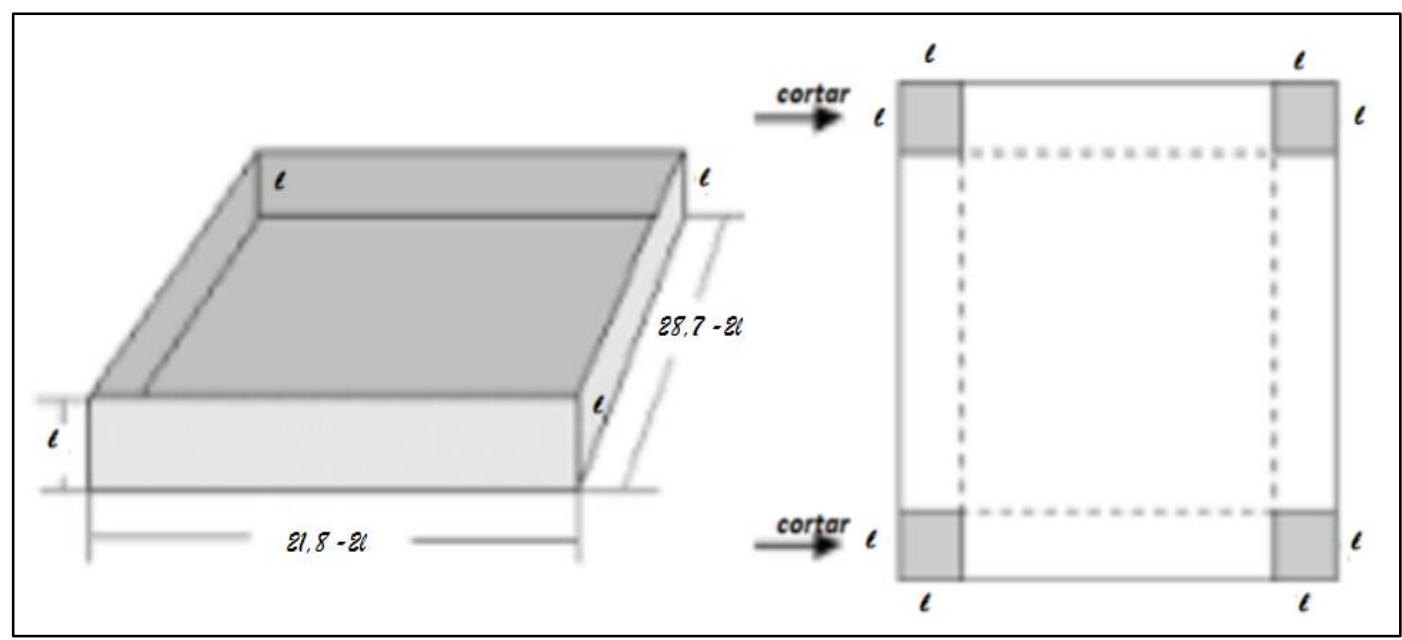

Figura 1 - Construcción de una caja sin tapa con una hoja de papel de $21.8 \mathrm{~cm} \times 28.7 \mathrm{~cm}$ Fuente: creación propia

Orientaciones $\left(\mathrm{O}_{\mathrm{i}}\right)$ : 
$O_{1}$ : Realiza el proceso de quitar cuadraditos de lado l en las esquinas de la caja, con varias hojas hasta encontrar la que tenga el volumen máximo.

$\mathrm{O}_{2}:$ ¿Cuáles deben ser las dimensiones de la caja, si se quiere que su volumen sea máximo?

$O_{3}$ : Encuentra el mayor número de representaciones posibles que representen el volumen de la caja.

$\mathrm{O}_{4}$ : Busca entre las representaciones del volumen de la caja, elementos equivalentes, y establece un paralelo entre ellos.

$O_{5}$ : Encuentra el mayor número de representaciones posibles que representen el área lateral de la caja.

$O_{6}$ : Busca entre las representaciones del área lateral de la caja, elementos equivalentes, y establece un paralelo entre ellos

En la elaboración de los planes de clases los estudiantes identificaron y describieron adecuadamente el contexto institucional, los indicadores de logro y los objetivos de la clase, atendiendo lo sugerido por el Ministerio de Educación Nacional (2016). Además, en una discusión grupal verificaron si con las actividades propuestas por cada uno se cumplirían los objetivos propuestos; según Schoenfeld y Kilpatrick (2008), este tipo de reflexiones, si se hacen habitualmente, pueden llegar a ser el principal mecanismo para mejorar la propia práctica.

Sin embargo, a la hora de escoger los estándares básicos de competencias (Ministerio de Educación Nacional, 2006), seleccionaron cualquiera que nombrara las funciones, independiente de que estuvieran o no en estrecha relación con los objetivos y los indicadores de logros planteados previamente. Se encontró cierta limitación en los estándares básicos de competencias en Matemáticas vigentes en Colombia, pues, para algunos objetivos e indicadores de logros propuestos, no se encontró estándares apropiados, esto dificultó su uso como configuraciones epistémicas de referencia. Esta dificultad se superó utilizando algunos de los estándares propuestos por el National Council of Teachers of Mathematics (2000).

En las clases de todos los profesores en formación se implementaron diversas estrategias para hacer fluir el conocimiento común del contenido, y tuvieron buen desempeño en el manejo de los conocimientos básicos desarrollados. Además, usaron adecuadamente su conocimiento ampliado: destacaron la importancia del tema en niveles más avanzados y similar a lo reportado por Chinnappan y Thomas (2001), luego de encontrar la representación algebraica, ésta se utilizó como representación principal y, a partir de ahí, obtuvieron las otras representaciones que lograron producir.

Todos utilizaron más de dos registros semióticos de representación, con sus respectivas representaciones: figural, gráfica, analítico algebraica, analítico numérica, tabular y/o del lenguaje coloquial. Poder producir varias representaciones de un mismo objeto es fundamental para la comprensión en matemáticas, ya que según Villa-Ochoa (2015), si una persona no es capaz de hacer conversiones y/o tratamientos entre por lo menos dos representaciones del objeto 
estudiado, termina estudiando la representación que haya concebido en lugar del objeto que ésta representa. Al respecto, Pino-Fan et al. (2017) consideran que el contenido de una representación semiótica nunca debe confundirse con el objeto matemático que ella representa.

En relación con el conocimiento especializado del contenido, hicieron muy buenos intentos por integrar los contenidos a los contextos socioculturales (AKÉ, 2013), utilizaron como material didáctico la hoja tamaño carta, pero solamente la manipularon ellos, quitándole así, al estudiante la posibilidad de explotar y explorar sus potencialidades matemáticas, omitieron que, como dice Tünnermann (2011), el estudiante debe ser el protagonistas de su propio proceso de aprendizaje, si se quiere que pueda aprender a aprender y, así, seguir aprendiendo durante toda su vida.

Respecto a los elementos matemáticos primarios, usaron variadas definiciones del objeto matemático función (AMAYA; PINO- FAN; MEDINA, 2016), así como procedimientos algebraicos adecuados, haciendo generalizaciones usando lenguaje simbólico-literal. El tipo de proposiciones también fueron adecuadas, y los argumentos, con buenas explicaciones sin justificarlas. Un ejemplo de lo anterior puede apreciarse en la clase desarrollada por $\mathrm{P}_{(6) 3}$, cuando dice:

$\mathrm{P}_{(6) 3}$ : el mayor volumen lo obtenemos si tomamos como referencia de corte de las esquinas $4,09 \mathrm{~cm}$, luego podemos encontrar el dominio de la función Volumen, al igual que el rango y su representación algebraica, que resultó ser $V(x)=(21,8-2 x)(28,7-2 x) x$

Los elementos lingüísticos utilizados fueron mayoritariamente verbales, combinados con números, expresiones algebraicas, signos de agrupación y de operación. El lenguaje matemático utilizado fue coherente y adecuado. Sin embargo, similar a lo sucedido con el material didáctico, los registros y las representaciones semióticas, solo los produjeron sin analizarlos en profundidad. Un ejemplo de ello es que realizaban congruencias entre algunas de las representaciones, y aunque ya las habían producido y tenían una gama de éstas disponibles para establecer las conexiones que les permitieran promover la asignación de significado y sentido a la función, al relacionarla con elementos del contexto sociocultural, los poco que lo hicieron (14\%), solo lograron establecer congruencias entre algunas representaciones por pares (solo uno lo hizo con más de dos), pero ninguno llegó a analizar el sistema semiótico emergente completo.

Esta dificultad para articular los elementos de las representaciones, y asignarle significado y sentido al objeto matemático estudiado, es un aspecto del conocimiento especializado del contenido indispensable para la enseñanza (AKÉ, 2013) comprensiva del objeto función. En este sentido, Even (1990) considera que la capacidad de identificar y 
reproducir un mismo objeto matemático en diferentes representaciones, y la flexibilidad de las conversiones entre éstas, son procesos cruciales en el aprendizaje de las matemáticas, ya que facilita a los estudiantes la visualización de relaciones ricas, y el desarrollo de una comprensión muy profunda de los conceptos. Pero también, que la falta de relaciones ricas y de conexión entre representaciones parece impedir que los profesores en formación puedan realizar conversiones entre diferentes representaciones de una función. Así, estas limitaciones en el conocimiento especializado del profesor en formación podrían afectar los procesos que en un futuro se orienten.

La falta de coordinación de las representaciones producidas podría resultar problemático, teniendo en cuenta que la comprensión en matemáticas necesita de la coordinación y el funcionamiento sinérgico de varios registros (DUVAL, 2017) y, porque el establecimiento de conexiones entre los elementos de varias representaciones de un mismo objeto facilita al profesor de matemáticas hacer un análisis ontosemiótico entre los elementos de dichas representaciones (DUVAL, 2004).

Otro aspecto que denotó limitaciones en el conocimiento especializado del contenido, de este grupo de profesores en formación, fue poder identificar y luego tratar las dificultades de los estudiantes en sus intentos de solución de la tarea. Aspecto que les hubiera permitido articular las teorías psicológicas de aprendizaje con la práctica (DOLORES, 2013), para utilizar los errores y dificultades de los estudiantes en beneficio de su propio proceso de aprendizaje y, en última, responsabilizarlos de éste.

Un caso muy especial fue la clase realizada por $\mathrm{P}_{(8) 8}$ quien abordó tanto la función Volumen, como la de Área lateral (ver Figura 2). Además, solo él analizó los intervalos de crecimiento/decrecimiento de dichas funciones, y mencionó que la gráfica del área lateral, no cruza el eje $x$ (eje 1 en este caso), y el significado que esto tiene en el marco de la situación: Esto se evidencia cuando dice:

$\mathrm{P}_{(8) 8}$ : pues lo que le falta a la gráfica para alcanzar el eje $x$ corresponde a la parte de hoja que no se cortó del largo, por ser mayor el largo que el ancho y, por tanto, en todas las hojas, al construir la caja, siempre quedó un rectángulo de la parte central de cada hoja

La articulación que $\mathrm{P}_{(8) 8}$ hace entre los elementos del sistema semiótico emergente fue algo atípico en el grupo, pues, la dificultad para relacionar los elementos de la relación funcional propuesta, con el uso que se hace de la noción función a nivel social, fue uno de los principales conflictos epistémicos encontrados en este estudio, que refuerza lo reportado por Godino, Bencomo y Wilhelmi (2006) y Font (2011). 


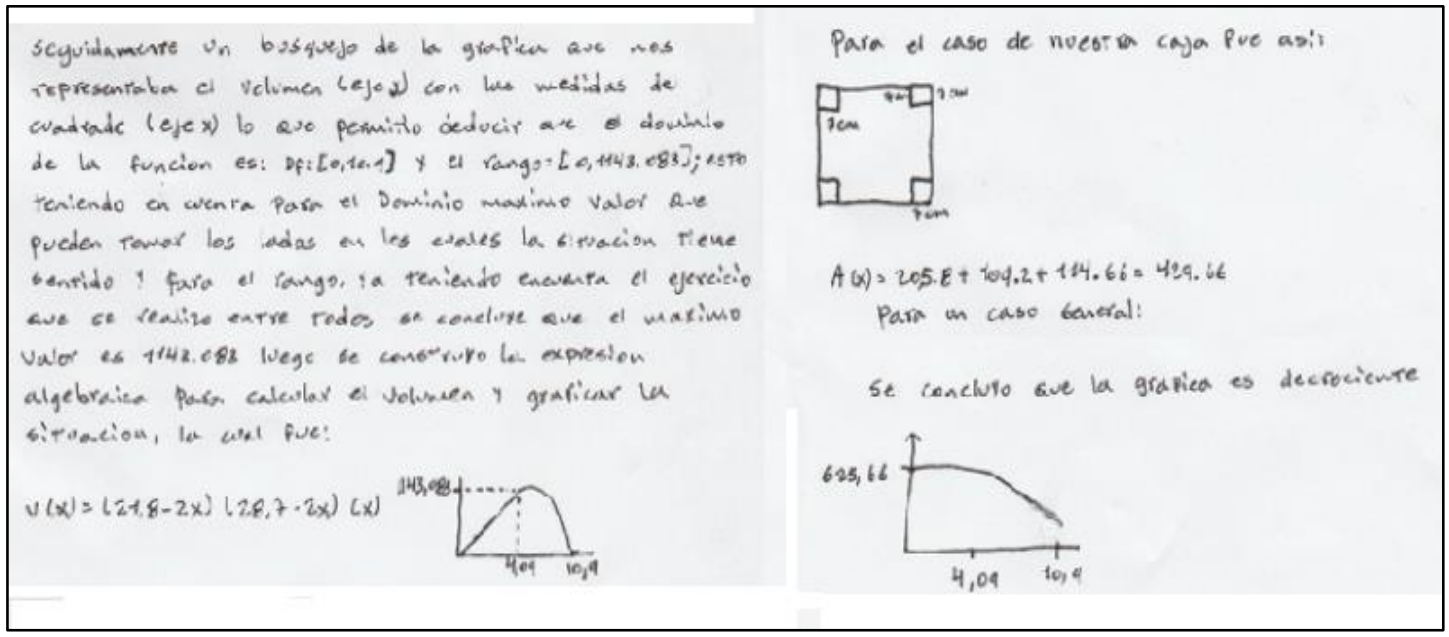

Figura 2 - Parte del manuscrito de $\mathrm{P}_{(\mathbf{8}) 8}$ mostrando la representación gráfica del área lateral de las cajas construidas por él Fuente: creación propia

Un hecho que pudo impedir que los estudiantes hicieran un análisis más adecuado del sistema semiótico emergente fue considerar los valores del dominio como discretos, es decir, que los valores $l$ a cortar tuvieran incrementos de uno en uno, y que todos fueran valores enteros. Esto pudo impedir el establecimiento de buenas conexiones entre los elementos visibles en distintas representaciones, que permitieran asignar significado y sentido al objeto matemático función.

Este aspecto fue una limitante para ambos grupos de profesores en formación. Por ejemplo, de haber hecho, con algún detalle, el análisis de la gráfica de la función Área lateral, hubieran podido caer en cuenta que su rango es $R_{A}=[150.42,625.66]$ y no $R_{A}=$ $[0,625.66]$ como lo visionaron muchos de ellos (64\%). Estos conflictos donde se confundía la letra como magnitud con la letra como variable generalizada (FONT, 2011) fueron muy difíciles de reconocer y de tratar en los profesores en formación. Para el caso, no pudieron armonizar sus conocimientos - ampliado y especializado - del contenido en beneficio de su conocimiento común.

Esto se evidenció, en los errores cometidos al tratar de hacer transposiciones didácticas para articular los elementos de las representaciones producidas, con elementos correspondientes del registro fenomenológico. Además, formalizaron el dominio y el rango de las funciones de variable generalizada asociadas, en lugar del de las relaciones funcionales estudiadas.

Lo anterior suele ser problemático si se quieren profesores idóneos, que orienten la clase de matemáticas eficientemente. Para ello se requiere que los conocimientos - ampliado y especializado - del contenido, en quien orienta el proceso, estén armonizados en pro del conocimiento común. Por lo que el profesor debe ser capaz de reproducir y poder transformar representaciones del objeto que estudie, articulando y analizando sus elementos. 
Este proceso permite percibir las implicaciones de la integralidad de la faceta epistémica, como fundamento de los conocimientos didáctico-matemáticos de un profesor, capaz de dar sentido a los objetos matemáticos que enseña, al relacionarlos con elementos del contexto sociocultural donde desarrolla su práctica. Al respecto Godino, Batanero y Font (2003, p.66) consideran que "no es posible dar sentido pleno a los objetos matemáticos si no se relacionan con los problemas de donde han surgido".

En relación con las actividades de afianzamiento y profundización, propuestas en clase, los profesores en formación, similar a lo reportado por Aké (2013), solo realizaron algunas modificaciones a las situaciones resueltas previamente, conservando la misma estructura y solo cambiando algunos datos. La dificultad en el planteamiento de este tipo de actividades denota, en el profesor, problemas en el entendimiento y razonamiento exclusivo de la enseñanza, más allá del conocimiento matemático en sí que se está enseñando (SGRECCIA; MASSA, 2012), pero que según Ball, Thames y Phelps (2008) requiere del profesor gran habilidad matemática y comprensión de lo que enseña. Además, en el trabajo con funciones, la elección de tareas que permitan la articulación de los problemas con los significados matemáticos es crucial para promover la idoneidad epistémica y cognitiva del aprendizaje significativo de este concepto (BUENO; PÉREZ, 2018).

\section{Conclusiones}

El estado de desarrollo de la faceta epistémica del conocimiento didáctico-matemático de este grupo de profesores en formación sobre funciones, evidencia algunas deficiencias formativas: mientras los conocimientos común y ampliado muestran un buen estado de desarrollo, su conocimiento especializado del contenido muestra serias limitaciones.

Este tipo de limitaciones, en los profesores en formación, invita a una revisión de los procesos instructivos del programa que los forma, que permita incorporar elementos que faciliten la coordinación de los sistemas semióticos emergentes, producidos en el proceso de estudio. Esto porque, según Wilhelmi, Godino y Lasa (2015), no basta con que los profesores hagan un uso operativo de las funciones, además, necesitan del conocimiento especializado del contenido que permita comprender potenciales conflictos de aprendizaje y diseñar situaciones que lleven a resolverlos.

Los profesores en formación lograron identificar los elementos y características de una función y reprodujeron varias de sus representaciones, pero presentaron dificultades al coordinar sus elementos y al relacionarlos con elementos correspondientes del contexto 
sociocultural, donde pudieran asignarles significado y sentido. Esto hace pensar que dichas dificultades pudieran tener su génesis en algunas limitaciones en su conocimiento especializado del contenido. Esta dificultad pudiera superarse, adecuando procesos de enseñanza y aprendizajes donde los profesores en formación logren producir diferentes representaciones de una misma relación funcional y establecer congruencias con elementos de la representación fenomenológica (AMAYA; PINO- FAN; MEDINA, 2016).

Entre los principales conflictos epistémicos de este grupo de profesores en formación, en el trabajo con funciones están: (a) la confusión de la letra como magnitud, con la letra como variable generalizada, ya que, al formalizar el dominio y el rango de una relación funcional, comunicaron los de las funciones de variable generalizada asociadas. (b) Poder asociar los elementos de una relación funcional, con el uso que se hace de la noción función a nivel social, por lo que, como en AMAYA; PINO- FAN; MEDINA (2016), parece haber un distanciamiento bien marcado entre la comprensión de la noción función a nivel escolar y su necesidad de uso consciente a nivel social.

Se concluye sobre la necesidad de seguir implementando procesos investigativos, que profundicen en los conocimientos didáctico-matemáticos de un profesor sobre funciones, y los niveles de abstracción adecuados para que este concepto pueda ser enseñado. En este sentido, se sugiere el análisis de los sistemas semióticos emergentes, que de una función se logren producir, como fundamento para que los profesores en formación, puedan ayudar a sus estudiantes a darle significado cuando la enseñan.

\section{Referencias}

AKÉ, L. Evaluación y desarrollo del razonamiento algebraico elemental en maestros en formación. Granada: Universidad de Granada, 2013.

AMAYA, T.; PINO-FAN, L.; MEDINA, A. Evaluación del conocimiento de futuros profesores de matemáticas sobre las transformaciones de las representaciones de una función. Revista Educación Matemática, Ciudad de México. v. 28, n. 3, p. 111-144, dic. 2016.

APÓSTOL, T. Calculus. (Tomo I). Barcelona: Reverté, 1985.

BALL, D.; HILL, H.; BASS, H. Knowing mathematics for teaching: Who knows mathematics well enough to teach third grade, and how can we decide? American Educator Chicago. v. 29, p. 14-22, 2005 .

BALL, D.; THAMES, M.; PHELPS, G. Content Knowledge for Teaching. What Makes It Special? Journal of Teacher Education, Michigan. v. 59, n. 5, p. 389-407, 2008.

BORJI, V.; SÁNCHEZ, A. An Exploratory Analysis of the Representations of Functions in the University Entrance Exam in Spain and Iran. Eurasia - Journal of Mathematics, Science and Technology Education London. v. 15, n. 8, p. 1-12, 2019. 
BREDA, A., PINO-FAN, L., \& FONT, V. Meta didactic-mathematical knowledge of teachers: criteria for the reflection and assessment on teaching practice. Eurasia - Journal of Mathematics, Science and Technology Education, London. v. 13, n. 6, p. 1893-1918, 2017.

BUENO, S.; PÉREZ, O. Prácticas actuales de la idoneidad epistémica y cognitiva del concepto función real de una variable real en carreras de ingeniería. Revista Educación Matemática, Ciudad de México. v. 30, n. 2, p. 202-231, ago. 2018.

CHINNAPPAN, M.; THOMAS, M. Prospective teachers' perspectives on function representation. In: ANNUAL CONFERENCE OF THE MATHEMATICS EDUCATION RESEARCH GROUP OF AUSTRALASIA, 24., 2001, Sydney. Proceedings of the 24th Conference of the International Group for the Psychology of Mathematics Education. Sidney: Merga, 2001. p. 155-162.

DOLORES, C. La variación y la derivada. 2. ed. México: Ediciones Díaz de Santos. 2013.

DUVAL, R. Semiosis y pensamiento humano: Registros semióticos y aprendizajes intelectuales. 2. ed. Cali: Universidad del Valle, 2017.

DUVAL, R. Los problemas fundamentales en el aprendizaje de las matemáticas y las formas superiores del conocimiento. Cali: Universidad del Valle, 2004.

ERTMER, P.; OTTENBREIT-LEFTWICH, A. Teacher Technology Change: How Knowledge, Confidence, Beliefs, and Culture Intersect. Journal of Research on Technology in Education. Miami. v. 42, n. 3, p. 255-284, 2010.

EVEN, R. Subject-Matter Knowledge for Teaching and the case of functions. Educational Studies in Mathematics, Dordrecht. v. 21, p. 521-544, 1990.

FONT, V. Las funciones y la competencia disciplinar en la formación docente matemática. UNO: Revista de Didàctica de las Matemáticas, Barcelona. v. 56, p. 86-94, 2011.

GODINO, J. Categorías de Análisis de los conocimientos del Profesor de Matemáticas. Revista iberoamericana de educación matemática, Buenos Aires. n. 20, p. 13-31, 2009.

GODINO, J. D.; BATANERO, C. Significado institucional y personal de los objetos matemáticos. Recherches en Didactique des Mathématiques, Grenoble, v. 14, n. 3, p. 325-355, 1994.

GODINO, J.; BATANERO, C.; FONT, V. Un enfoque ontosemiótico del conocimiento y la instrucción matemática. ZDM, Barcelona. v. 39, n. 1-2, p. 127-135, 2007.

GODINO, J.; BATANERO, C.; FONT, V. Fundamentos de la enseñanza y el aprendizaje de las matemáticas para maestros. Granada: Universidad de Granada. 2003.

GODINO, J.; BENCOMO, C.; WILHELMI, M. Análisis y valoración de la idoneidad didáctica de procesos de estudio de las matemáticas. Paradigma, Maracay. v. 27, n. 2, p. 221-252, 2006.

GODINO, J.; GIACOMONE, B.; BATANERO, C.; FONT, V. Enfoque Ontosemiótico de los Conocimientos y Competencias del Profesor de Matemáticas. Bolema, Rio Claro, v. 31, n. 57, p. 90113, abr. 2017.

GROSSMAN, P. The making of a teacher: Teacher knowledge and teacher education. Nueva York: Teachers College Press. 1990.

HACIOMEROGLU, G. Prospective secondary teachers' subject matter knowledge and pedagogical content knowledge of the concept of function. 2005. Thesis (Doctorate in Philosophy) - Department of 
Middle \& Secondary Education, Florida State University, Florida, 2006.

HILL, H.; BALL, D.; SCHILLING, S. Unpacking pedagogical content knowledge: Conceptualizing and measuring teachers' topic-specific knowledge of students. Journal for Research in Mathematics Education, Reston. v. 39, n. 4, p. 372-400, jul. 2008.

HITT, F. Dificultades en el aprendizaje del cálculo. Décimo primer Encuentro de Profesores de Matemáticas del Nivel Medio Superior. Morelia: Universidad Michoacana de San Nicolás de Hidalgo, 2003.

HITT, F.; MORASSE, C. Pensamiento numérico-algebraico avanzado: construyendo el concepto de covariación como preludio al concepto de función. Electronic Journal of research in educational psychology, Almería. v. 7, n. 17, p. 243-260, abr. 2009.

MINISTERIO DE EDUCACIÓN NACIONAL. Derechos básicos de aprendizaje. Bogotá: Panamericana Formas e Impresos S.A, 2016.

MINISTERIO DE EDUCACIÓN NACIONAL. Estándares básicos de competencias en Lenguaje, Matemáticas, Ciencias y Ciudadanas. Bogotá: Ministerio de Educación Nacional, 2006.

NATIONAL COUNCIL OF TEACHERS OF MATHEMATICS. Principios y Estándares para la Educación Matemática. Traducción al español, Sociedad Andaluza de Educación Matemática Thales. Sevilla: Proyecto Sur, 2000.

PEIRCE, C. The Fixation of Belief. Popular Science Monthly, Nueva York. v. 12, n. 1, p. 1-15, 1877.

PINO-FAN, L. Evaluación de la faceta epistémica del conocimiento didáctico - matemático de futuros profesores de bachillerato sobre la derivada. 2013. Tesis (Doctorado en Didáctica de las matemáticas) - Departamento de Didáctica de la Matemática, Universidad de Granada. Granada: 2013.

PINO-FAN, L.; ASSIS, A.; CASTRO, W. Towards a Methodology for the Characterization of Teachers' Didactic-Mathematical Knowledge. Eurasia - Journal of Mathematics, Science and Technology Education, London. v. 11, n. 6, p. 1429-1456, dec. 2015.

PINO-FAN, L.; GODINO, J. Perspectiva ampliada del conocimiento didáctico - matemático del profesor. Paradigma, Maracay. v. 36, n. 1, p. 87-109, jun. 2015.

PINO-FAN, L.; GUZMÁN, I.; FONT, V.; DUVAL, R. Analysis of the Underlying Cognitive Activity in the Resolution of a Task on Derivability of the Absolute-Value Function: Two Theoretical Perspectives. PNA, Granada. v. 11, n. 2, p. 97-124, nov. 2017.

PINO-FAN, L., PARRA, Y. \& CASTRO, W. Significados de la función pretendidos por el currículo de matemáticas chileno. Magis - Revista Internacional de Investigación en Educación, Bogotá. v. 11, n. 23, p. 201-220, ene. 2019.

REY, G.; BOUBÉE, C.; SASTRE, P.; CAÑIBANO, A. Ideas para Enseñar. Aportes didácticos para abordar el concepto de función. Revista iberoamericana de educación matemática, Buenos aires. n. 20 , p. $153-162$, dic. 2009.

ROJAS, P. Objetos matemáticos, representaciones semióticas y sentidos. Revista Enseñanza de las ciencias, Barcelona. v. 33, n. 1, p. 151-165. 2015.

SCHOENFELD, A.; KILPATRICK, J. Towards a theory of profiency in teaching mathematics. In: TIROSH, D.; WOOD, T. (Ed.). Tools and Processes in Mathematics Teacher Education Rotterdam: Sense Publishers, 2008. p. 321-354. 
SGRECCIA, N.; MASSA, M. Conocimiento especializado del contenido de estudiantes para profesor y docentes noveles de matemáticas. Revista Educación Matemática, Ciudad de México. v. 24, n. 3, p. 33-66, dic. 2012.

SHULMAN, L. Conocimiento y enseñanza: fundamentos de la nueva reforma. Revista de currículo y formación del profesorado, Granada. v. 9, n. 2, p. 1-30, 2005.

SHULMAN, L. Knowledge and teaching: Foundations of the new reform. Harvard Educational Review, Cambridge. v. 57, n. 1, p. 1-22, 1997.

TÜNNERMANN, C. El constructivismo y el aprendizaje de los estudiantes. Universidades, Ciudad de México. n. 48, p. 21-32, 2011.

VILLA-OCHOA, J. Modelación matemática a partir de problemas de enunciados verbales: un estudio de caso con profesores de matemáticas. Magis - Revista Internacional de Investigación en Educación, Bogotá. v. 8, n. 16, p. 133-148, 2015.

WILHELMI, M.; GODINO, G.; LASA, A. Significados conflictivos de ecuación y función en estudiantes de profesorado de secundaria. In: GONZÁLEZ, M.; CODES, M.; ARNAU, D.; ORTEGA, T. (Ed.). Investigación en educación matemática. Salamanca: Sociedad Española de Investigación en Educación Matemática, 2015. p. 573-582. 